\title{
Node Failures and Space Complexity Variations in Step Topology under Distributed Environment
}

\author{
Taskeen Zaidi \\ Research Scholar, Deptt. of Computer Science \\ Babasaheb Bhimrao Ambedkar University \\ Vidya Vihar, Rae Bareli, Lucknow
}

\author{
Vipin Saxena \\ Professor, Deptt. of Computer Science \\ Babasaheb Bhimrao Ambedkar University \\ Vidya Vihar, Rae Bareli Road, Lucknow
}

\begin{abstract}
During the past years, distributed computing approach is most popular approach among the other computing approaches. In this approach, the execution time of the processes has been reduced and the setup cost of distributed systems is very low. The process is defined as subtasks, subroutines, macro's etc. In the present work, a static interconnection of the computer systems is defined and called as the static step topology. By the use of this topology, $\mathrm{N}$ numbers of hand-held devices and other computer systems may be attached. As the size of network increases, the space complexities are computer by varying the length of the cable segments. Node failures are also computed in the static step topology. A Unified Modeling Language (UML) is used to demonstrate the dynamic execution of process in step topology and represented through sequence diagram.
\end{abstract}

\section{Keywords}

Distributed approach, static step topology, space complexity, node failures and UML.

\section{INTRODUCTION}

Distributed system is a collection of the autonomous different devices called as smart devices. These devices can be connected by the use of static and dynamic topologies and these are well defined in a famous book written by Hwang [1]. Various kinds of the distributed algorithms for execution of process under mutual exclusion are available in [2]. Milenkovic [2] has also explained necessary conditions for accessing critical sections for allocation of resources to the processes. Before solution of the research problem, modeling aspects are necessary to judge the right design at the early stages of development. In 2002, first time, Pllana and Fahringer [3] have suggested UML profiles for measuring the performance metrics of distributed and parallel applications. The Unified Modeling Language (UML) has been developed by OMG (Object Management Group), which is widely accepted by software professionals for modeling various research problems. It's features and versions are well explained in [4-5]. Zaidi and Saxena [6] has designed a step topology for static interconnection of devices under distributed environment in which $\mathrm{N}$ number of devices can be connected by increasing the length of cable segments. Further, they studied the impact of Information Communication Technology (ICT) on National Knowledge Network (NKN) [7]. Its benefits are well explained and a case study for percentage utilization of NKN network is also studied in the paper. Modeling of step and bus topology has done by Zaidi and Saxena [8] in which both topologies are compared with activities. Diameters are also computed by varying the nodes which is represented in form of table and graphs. Zaidi and Saxena [9] have also studied link failures and bypass link failures in the Mobile Adhoc Network (MANET) which is arranged through step topology and allocation of resources are also described by the use of UML. The ladder topology is well defined in the reference [10]. Description on Miniaturized transmission lines based on hybrid lattice-ladder topology is available in [11].

\section{BACKGROUND}

\subsection{Process and Thread}

A task or process is defined as a collection of lines of code called as subprogram, macro, and subroutine or defined as a unit of work to be executed by the use of Processor. Processing Element Unit called as PEC, which is attached to the processor; is fully responsible to execute the process. Process has attributes like Process_size, Process_id, Process_in_time, Process_out_time, Process_type, Process_cardinality and Process_priority. These all are controlled by created, delete, update, join, suspend, synchronize and are represented by methods Process_created, Process_delete, Process_update, Process_join, Process_suspend, Process_synchronize. On the other hand, Threads control a process that consists of number of subprograms. The threads run concurrently and called as light weight process; follow the sequential flow of instructions. The threads run simultaneously in the process and access the objects in concurrent manner. They have number of attributes Thread_id, Thread_size, Thread_name, Thread_priority and perform operations like Thread_synchronize, Thread_resume, Thread_start, Thread_terminate, Thread_interrupt, Thread_join, Thread_stop and Thread_suspend.

\subsection{Distributed System}

A distributed system is defined as collection of autonomous devices like computer systems, mobile computer systems, ipads, PDA's or any handheld devices. A network of interconnection of these systems is called as National Knowledge Network (NKN) as per India Nation, which has been implemented under Next Generation Network (NGN) services. These systems cann't share the global clock while they can share both local as well as remote resources. Each device as said above has variation in the hardware specifications. The devices under the distributed systems are interacted with the help of message passing techniques. A distributed system under NKN is shown below in fig. 1 . 


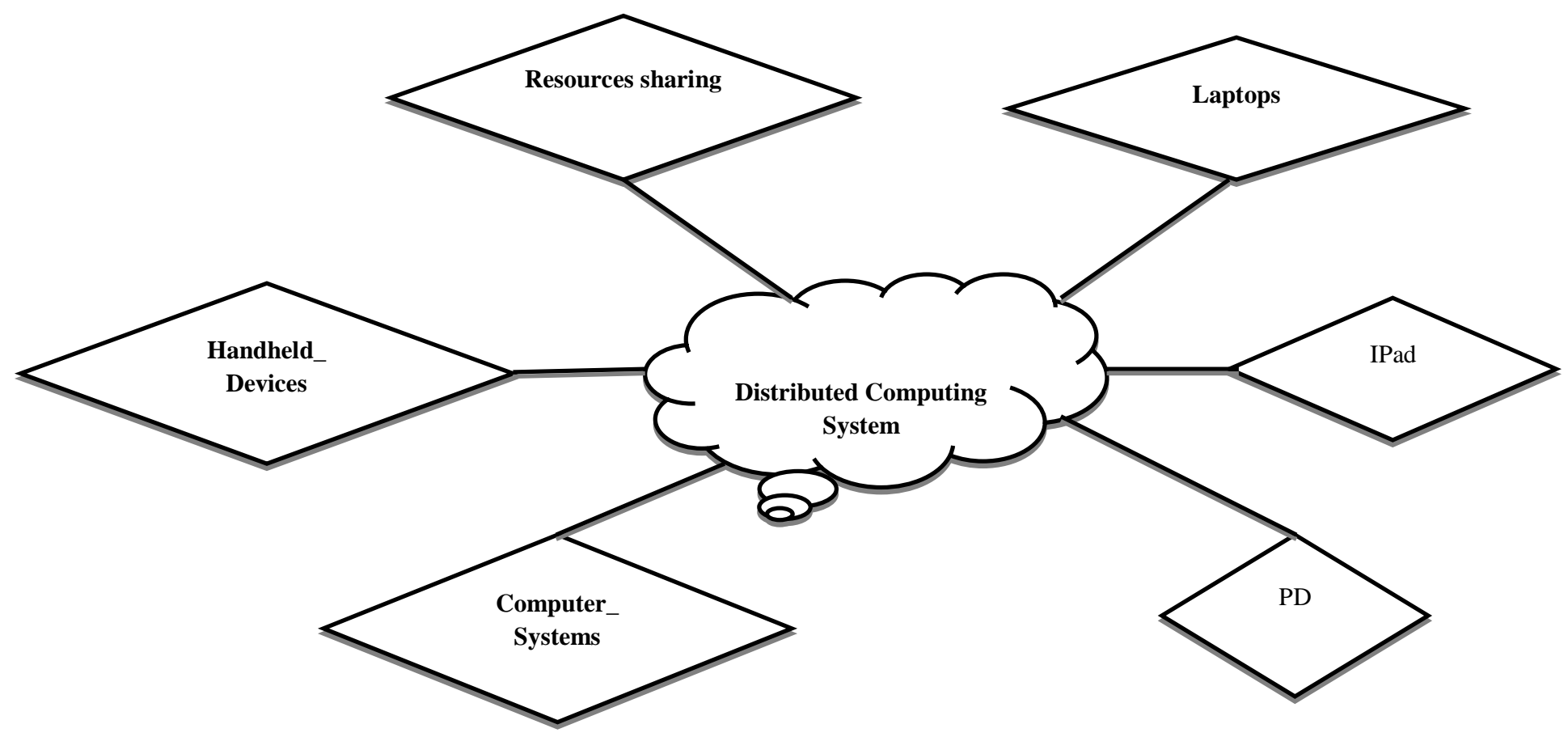

Figure 1: A Distributed System

\subsection{Communication Lines}

The communications under the distributed system are of the four categories namely one to one, one to many, many to one and many to many communications as supported by the Process_cardinality attribute defined under the definition of Process class. These processes are synchronized by using the message passing techniques. The processes use the resources, one by one for uniprocessors while they can access resourcesSends concurrently on more than one processor. If one process is inside the critical section then no other process is allowed to use the critical section. Different ways of communication lines are shown below in the following figs. 2-4 for one to one, one to many or many to one, many to many, respectively. In these figures Ack shows the acknowledgement of the received information.

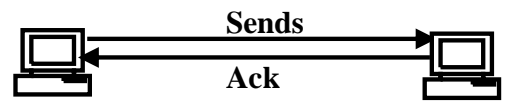

Figure 2: One to One Communication
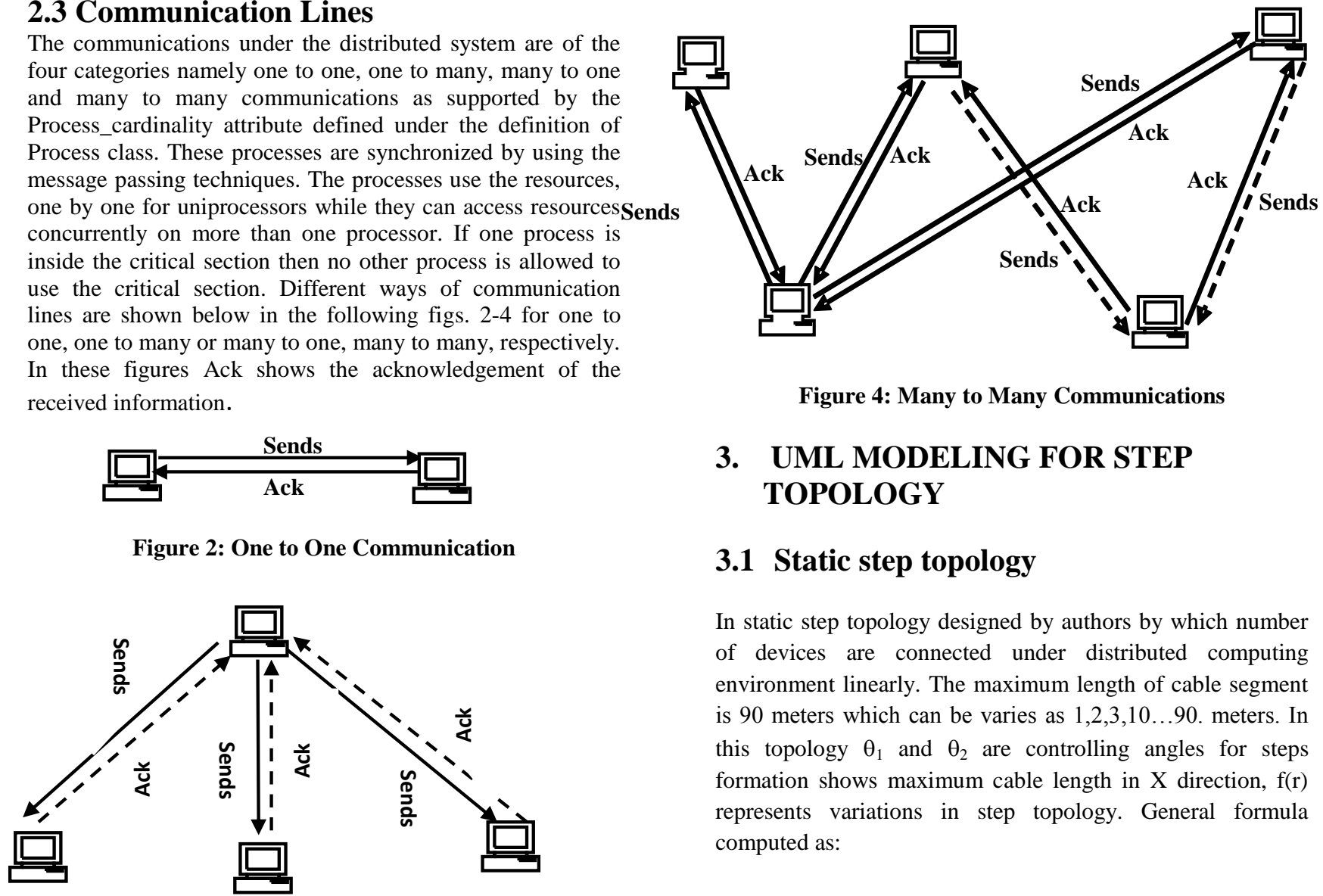

Figure 4: Many to Many Communications

\section{UML MODELING FOR STEP TOPOLOGY}

\subsection{Static step topology}

In static step topology designed by authors by which number of devices are connected under distributed computing environment linearly. The maximum length of cable segment is 90 meters which can be varies as $1,2,3,10 \ldots 90$. meters. In this topology $\theta_{1}$ and $\theta_{2}$ are controlling angles for steps formation shows maximum cable length in $\mathrm{X}$ direction, $\mathrm{f}(\mathrm{r})$ represents variations in step topology. General formula computed as:

Figure 3: One to Many Communication or Many to One Communication 
$f(r)=1+\beta_{1} r \quad 0<r<\alpha \ldots \quad(i)$

$=1+\sum \beta_{i}\left(r-\alpha_{i+1}\right) \quad \alpha<r<1$ and $i=1(1)(N-1) \ldots(i i)$

where, $\beta_{1}=\tan \theta_{1}, \beta_{2}=\tan \theta_{2} \ldots \beta_{N}=\tan \theta_{N}$

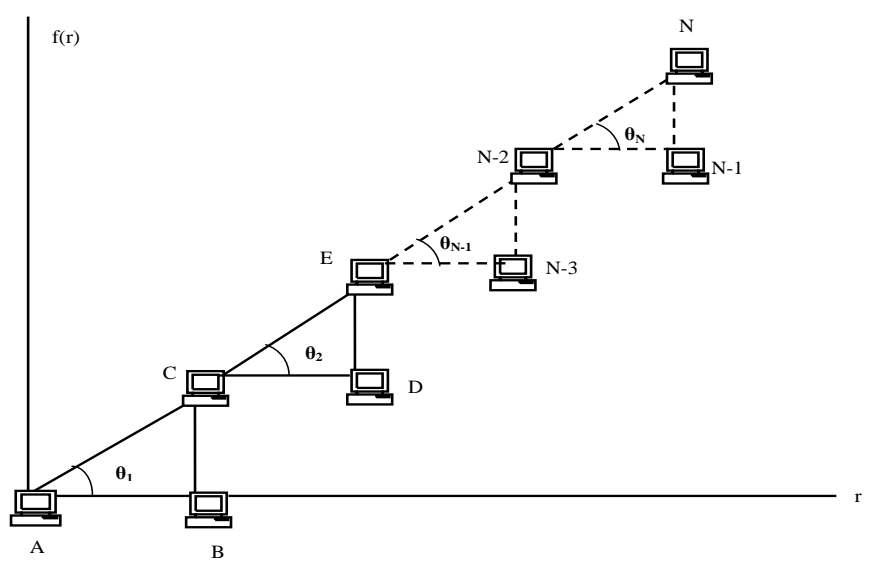

Figure 5: A Static Step Topology

\subsection{UML Sequence diagram}

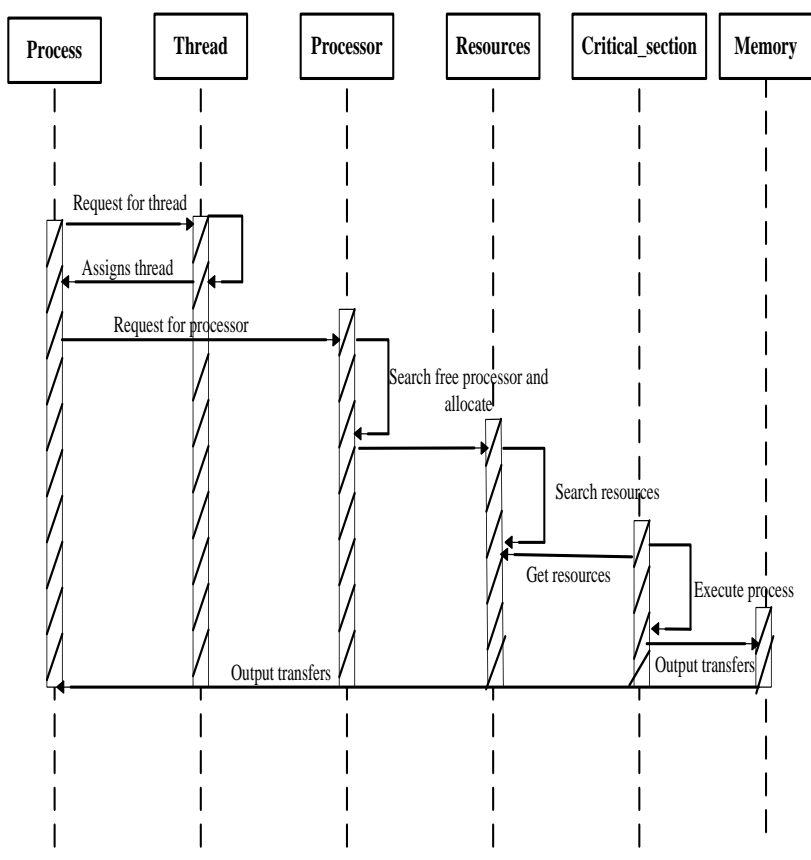

Figure 6: UML Sequence Diagram for Execution of Process

The above diagram shows dynamic behavior of the system for execution of the processes under distributed environment for the step topology. It consists of six objects which have life lines represented by the vertical lines. Initially, threads are assigned to the processes and then processes check the free processors, if the processor is free then after getting the resources, it can be executed in the critical section. After the completion of the execution of the process, the results are stored inside the memory and transferred to the process.

\section{Space Complexity Variations in Step Topology}

Case 1: Uniform Variation in Step Topology

For the uniform variations, let us consider as $\theta_{1}=\theta_{2}=45^{\circ}$ for which $\tan 45^{\circ}=1, \beta_{1}=\beta_{2}$, and in this case $\mathrm{f}(\mathrm{r})=1+\mathrm{r}$ supports linear variations. One can say that the step topology will move with the uniform angle. The computed result for this case is computer below in the following table 1 . The space complexity is of the $\mathrm{O}(\mathrm{r})$ which is linearly varying.

Table 1. Linear Variations in Step topology

\begin{tabular}{|c|c|}
\hline $\mathbf{r}$ & $\mathbf{f}(\mathbf{r})$ \\
\hline 10 & 11 \\
\hline 20 & 21 \\
\hline 30 & 31 \\
\hline 40 & 41 \\
\hline 50 & 51 \\
\hline 60 & 61 \\
\hline 70 & 71 \\
\hline 80 & 81 \\
\hline 90 & 91 \\
\hline
\end{tabular}

\section{Case 2: Mixed Variation in Step Topology}

For the mixed variations in the step topology, let us consider as $\theta_{1}=45^{\circ}, \theta_{2}=30^{\circ}$ for which $\tan \theta_{1}=1, \tan \theta_{2}=1 / \sqrt{ } 3$ and $\alpha=$ 50 meter. The computed results for double linear mixed variations are given below in the following table 2 . The results show the space complexity which is increasing in comparison of the uniform variation after $\mathrm{r}=50$, but the order of space complexity will remain same as $\mathrm{O}(\mathrm{r})$.

Table 2. Mixed Variations in Step topology

\begin{tabular}{|c|c|}
\hline $\mathbf{r}$ & $\mathbf{f}(\mathbf{r})$ \\
\hline 10 & 11 \\
\hline 20 & 21 \\
\hline 30 & 31 \\
\hline 40 & 41 \\
\hline 50 & 51 \\
\hline 60 & 67 \\
\hline 70 & 84 \\
\hline 80 & 98 \\
\hline 90 & 114 \\
\hline
\end{tabular}

\section{Node failures in step topology}

The static interconnection of computer systems is of fixed category therefore every static topology proposed in literature 
has some advantages and disadvantages. To explain the node failures in the proposed topology, let us consider the five computer systems A, B, C, D and E as shown in the fig.5. The drawbacks in the form of failure are summarized below in table 3. If the link between A-C fails then it will automatically convert into the bus topology like A-B-C and follow the advantages and disadvantages of bus topology.

Table 3. Node Failures in Step Topology

\begin{tabular}{|c|c|c|}
\hline Sl. No. & Link failure & $\begin{array}{c}\text { Node } \\
\text { failure }\end{array}$ \\
\hline 1 & A-E & C \\
\hline 2 & A-B,A-C & B \\
\hline 3 & A-C & C \\
\hline 4 & A-B,A-C & A \\
\hline 5 & C-D,C-E & C \\
\hline
\end{tabular}

\section{CONCLUSIONS}

From the above work, it is concluded that designed static step topology is very efficient for connecting the $\mathrm{N}$ number of devices. The NKN network is based on very powerful Endian operating system for keeping the records of the users attached on the computer networks. It is observed, as the numbers of connected nodes are increased, then the space complexities are also increasing for both kinds of variations in cable segments i.e. linear and mixed variations. The different kinds of the failures are also depicted for the step topology. Step topology si different from the bus which is linear and proposed topology is based on the step. In the bus topology if link fails between two nodes then they cannot communicate while in the step topology, they can communicate through the third node which is available on the next step. A very powerful Unified Modeling Language is used to show the dynamic behavior of the execution of the process under critical section.

\section{ACKNOWLEDGMENTS}

The authors are very thankful to University Grants Commission for providing financial assistance to the Department of Computer Science, Babasaheb Bhimrao Ambedkar University, Lucknow to carry out the above research work.

\section{REFERENCES}

[1] Hwang, K. "Advanced Computer Architecture: Parallelism, Scalability, Programmability", Fourteen Reprint, Tata Mc-Graw Hill Edition 2007.

[2] Milenkovic, M. "Operating Systems: Concepts and Design”, Tata McGraw Hill, 1987.

[3] Pllana, S. and Fahringer, T., "On Customizing the UML for Modeling Performance Oriented Applications". In $\langle\langle U M L\rangle>$, Model Engineering Concepts and Tools, Springer-Verlag., Dresden, Germany, 2002.

[4] Booch, G., Rumbaugh, J. and Jacobson, I., "The Unified Model-ing Language", User Guide, Addison Wesley, Reading, MA 1999.

[5] OMG, "Unified Modeling Language (UML)-Version 1.5", OMG document formal/2003-3-01, Needham MA (2003).

[6] Zaidi T. and Saxena, V., "Step Topology for Static Interconnection of Computer Systems under Distributed Environment", $3^{\text {rd }}$ World Conference on Information Technology organized by University of Bercelona, Nov. 14-17, 2012.

[7] Zaidi T. and Saxena, V., "National knowledge Network versus Information Communication Technology", In Proceeding of University Department of Mathematics, B.R.A. Bihar University, Muzzafarfur India, 11-12 Feb., 2012.

[8] Zaidi T. and Saxena, V., "Modeling Aspects for Step and Bus Topologies under Distributed Computing System", International Journal of Computer Applications, USA, Vol. 60(3), pp. 20-24, December 2012.

[9] Zaidi T. and Saxena, V., "Resources Allocation and Failures in Step Topology under Distributed Computing System", Journal of Software Engineering Applications, USA, to be published in Jan.2013.

[10] Shiramizu, N., Masuda, T., Nakamura, T. and Washio, K. (1968) "Scalable Transformer Model Based on Ladder Topological Equivalent Circuit for SiRFICs", doi: 10.1109/SMIC. 2010.5422954.

[11] Koohazadek, M. and Tamijani, A. (2010) "Miniaturized Transmission Lines Based on Hybrid Lattice-Ladder Topology", IEEE Transactions on Microwave Theory and Techniques, 58 (4). 